\title{
Avaliação farmacocinética de comprimidos contendo lamivudina e zidovudina em plasma humano
}

\author{
João Eudes do Nascimento ${ }^{1 *}$, Nereide Stela Santos Magalhães², Roseane Maria Ribeiro², \\ Adriana Pontes ${ }^{2}$, Antônio José Alves ${ }^{1}$
}

${ }^{1}$ Departamento de Ciências Farmacêuticas, Universidade Federal de Pernambuco, ${ }^{2}$ Laboratório de Imunopatologia Keizo-Asami

\section{*Correspondência:}

J. E. Nascimento

Departamento de Ciências

Farmacêuticas,

Av. Prof. Arthur Sá, s/n, Cidade

Universitária,

50740-520 - Recife - PE.

E-mail: eudes@ufpe.br
Este estudo se propõe a avaliar o perfil farmacocinético de comprimidos contendo lamivudina e zidovudina associados, produzidos pelo Laboratório Farmacêutico do Estado de Pernambuco - LAFEPE. O medicamento foi administrado em 10 voluntários sadios e foram observadas as concentrações plasmáticas dos dois anti-retrovirais durante 12 horas. A partir das concentrações plasmáticas foi possivvel encontrar os parâmetros farmacocinéticos de ambos os fármacos através de cálculos estatísticos. Os resultados obtidos foram comparados com aqueles resultantes de experimentos realizados por outros autores descritos neste trabalho, em que se verificou não haver diferenças significativas entre os valores encontrados e os descritos na literatura e que o fato dos anti-retrovirais estarem associados na mesma formulação não modificou o perfil cinético de nenhuma das substâncias.

\section{INTRODUÇÃO}

\section{Lamivudina}

Lamivudina (3TC) é um 2',3'-didesoxinucleosídeo, análogo sintético da citidina, com configuração absoluta $2 R, 5 S$ (Perry, Fauldes, 1997). É representada quimicamente como a forma negativa (cis) enantiomérica do 2'desoxi-3-tiacitidina -Perry, Fauldes, 1997; Johnson et al., 1999; Blaney et al., 1995.

Estruturalmente, a molécula de 3TC é a [2R-cis-(-)4amino-1-[2-(hidroximetil)-1-3-oxatiolan-5-il]-2(1Hpirimidina)] (Van Leeuwen et al.,1995; Robbins et al.,1998) (Figura 1).

\section{Unitermos}

- Farmacocinética

- Lamivudina

- Zidovudina
FIGURA 1 - Fórmula estrutural da lamivudina

A lamivudina, quando administrada por via oral, é rapidamente absorvida por difusão passiva na parede intestinal e possui biodisponibilidade sistêmica média absoluta em torno de $82 \%$ em adultos (Perry, Fauldes, 1997).

O tempo médio para se chegar à Concentração 
Sérica Máxima $\left(\mathrm{C}_{\max }\right)$ varia de 0,5 a 1,5 horas após a administração oral em dose única. Sendo administrada tanto por via oral como intravenosa, a lamivudina apresenta correlação linear nos parâmetros farmacocinéticos dosedependentes, com valores de $\mathrm{C}_{\max }$ e Área Sob a Curva (ASC) diretamente proporcionais à dose (Van Leeuwen et al., 1992; Johnson et al., 1999; Lewis et al., 1996).

Moore et al. (1999) analisaram a absorção do 3TC, quando administrado em combinação com a zidovudina (AZT), em doses de $150 \mathrm{mg}$ e $300 \mathrm{mg}$, respectivamente, e perceberam que não havia diferença significativa em relação à absorção deste mesmo fármaco, quando utilizado em monoterapia.

A molécula de lamivudina penetra livremente nos tecidos após atingir a circulação sistêmica. Após uma dose intravenosa que variou de 0,25 a $8 \mathrm{mg} / \mathrm{kg}$, o volume médio aparente de distribuição foi de aproximadamente 1,3 $\mathrm{L} / \mathrm{kg}$, indicando que o fármaco é distribuído em todo fluido corpóreo (Van Leeuwen et al., 1992). Após dose oral, o volume de distribuição aparente se mostrou dose-independente (Johnson et al., 1999). Esta ampla distribuição da lamivudina se deve, em parte à baixa, massa molecular (229) e sua ligação com as proteínas plasmáticas é baixa (36\%) (Johnson et al., 1999).

A lamivudina atinge o líquido céfalo-raquidiano (LCR) em pequenas quantidades. Adultos infectados com o Vírus da Imunodeficiência Humana Adquirida HIV (estudo com seis pacientes) apresentaram concentração de 94 a $328 \mu \mathrm{g} / \mathrm{L}$ de 3 TC no LCR a partir de uma dose de 8 a $20 \mathrm{mg} / \mathrm{kg} / \mathrm{dia}$ (Van Leeuwen et al., 1995).

A lamivudina não é significativamente metabolizada: aproximadamente de 5 a $10 \%$ da dose administrada é convertida em um metabólito trans-sulfóxido inativo e as concentrações deste ainda não foram identificadas em pacientes infectados com o HIV. A maior parte do fármaco é eliminada pela urina após 12 horas da administração da dose (Johnson et al., 1999; Perry, Fauldes, 1997).

Após administração oral de dose única de $0,25 \mathrm{~g}$ de lamivudina em 20 voluntários sadios a meia-vida $\left(\mathrm{t}_{1 / 2}\right)$ estimada foi de 2,5 horas (Van Leeuwen et al., 1992).

O Clearence renal $\left(\mathrm{Cl}_{\mathrm{R}}\right)$ do $3 \mathrm{TC}$ é maior que a filtração glomerular, indicando que a lamivudina é predominantemente eliminada por secreção tubular ativa renal (Van Leeuwen et al., 1995). Como a lamivudina é eliminada primariamente como fármaco não-transformado, um comprometimento renal altera significativamente a farmacocinética desta (Heald et al., 1996).

\section{Zidovudina}

A zidovudina (AZT) é uma pirimidina didesoxinu- cleosídica, análogo sintético da timidina. É representada quimicamente como a forma eritroisoméica (3'-azido-3'deoxitimidina) (Földes et al., 1993; Slusher et al., 1992; Langtry, Campoli-Richards,1989; Hedaya, Sawchuk, 1988) (Figura 2).

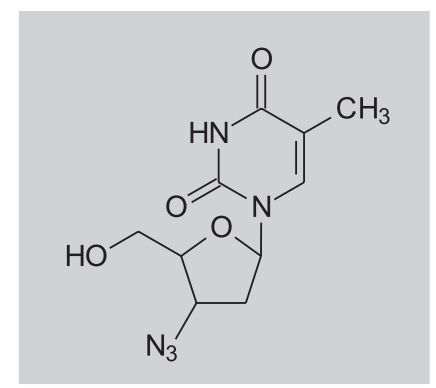

FIGURA 2 - Fórmula estrutural da zidovudina.

Após administração oral, o AZT é rapidamente e quase completamente absorvido, porém, aproximadamente $60 \%$ da dose estão disponíveis sistemicamente, o que sugere perda de aproximadamente $40 \%$ do fármaco, devido ao efeito de primeira passagem, uma vez que $90 \%$ da dose administrada pode ser recuperada na urina como metabólito ou fármaco inalterado (Klecker et al., 1987 Blum et al.,1988).

Os picos de concentração plasmática são atingidos após 0,5 a 1,5 horas após a administração oral. Doses de 2,5 e $10 \mathrm{mg} / \mathrm{kg}$ produziram $\mathrm{C}_{\max }$ de 1,5 a $2,0 \mu \mathrm{mol} / \mathrm{L}, 4,0$ a $6,0 \mu \mathrm{mol} / \mathrm{L}$ e 6,0 a $10 \mu \mathrm{mol} / \mathrm{L}$, respectivamente (Klecker et al., 1987), e cinética dose-independente foi observada quando comparada com administração intravenosa (Blum et al., 1988).

Quando AZT foi administrado em combinação com a lamivudina em um único comprimido, este se mostrou bioequivalente em relação à administração em separado (Moore et al., 1999).

Em humanos, AZT tem sido encontrado concentrado no sêmen de pacientes HIV-infectados com taxas variando de 1,3 a 20,4 vezes em relação ao plasma, 75 minutos após administração oral de $200 \mathrm{mg}$ (Henry et al., 1988). A maior penetração no líquido céfalo raquidiano foi observada por Klecker et al. (1987), 4 horas após dose oral de $10 \mathrm{mg} / \mathrm{kg}$, que, detectaram taxa de 1,35 vezes em relação ao plasma.

Em pacientes com Síndrome da Imunodeficiência Humana Adquirida (SIDA) o AZT distribui-se amplamente no corpo, com volume de distribuição médio de $1,4 \mathrm{~L} / \mathrm{kg}$, excedendo a água total do corpo por mais de $100 \%$ (Klecker et al., 1987).

O metabolismo do AZT foi analisado primeiramente em macacos por Good et al. (1986), isolando o 
metabólito 3'-azido-3'-desoxi-5' ' $\beta$-D-glicopiranosil timidina, recuperado na urina. Comparado com seu metabólito, uma quantidade maior de AZT é recuperada na urina de pacientes com SIDA (aproximadamente 3:1), após administração intravenosa ou oral do fármaco.

Em pacientes infectados com HIV tem-se observado meia-vida muito curta, 1,5 $\pm 0,35$ horas (Klecker et al., 1987) e 1,1 $\pm 0,3$ horas após administração oral de $250 \mathrm{mg}$ (Barry et al., 1994) e 1,50 \pm 0,24 horas após administração oral de 300 mg (Moore et al., 1999).

O clearence plasmático em 11 pacientes foi de 1,3 $\mathrm{L} / \mathrm{kg} / \mathrm{h}$, com clearence renal médio de $0,23 \mathrm{~L} / \mathrm{kg} / \mathrm{h}$, sugerindo que ocorre secreção tubular renal do AZT, já que a taxa excede a taxa de filtração glomerular normal (Klecker et al., 1987).

\section{Associação AZT/3TC}

O AZT foi o primeiro anti-retroviral aprovado pelo Food and Drug Administration (FDA), em 1987. Contudo, o seu uso prolongado promoveu o aparecimento de efeitos tóxicos, resistência viral ao fármaco e falha na terapêutica. $\mathrm{O}$ uso da monoterapia com AZT em casos avançados da doença promoveu resistência ao fármaco em seis meses, $\mathrm{o}$ mesmo ocorreu com a lamivudina em menor intervalo de tempo. Combinações de fármacos foram urgentemente requisitadas para conter a resistência e a replicação viral (Larder et al., 1995).

A lamivudina foi sintetizada com a finalidade de aumentar a segurança durante o longo prazo da terapia, modificando-se a estrutura da molécula 2'3'-didesoxicitidina (análogo sintético da citidina) com a substituição do metileno por um átomo de enxofre no carbono 3' do anel da ribose (Van Leeuwen et al., 1995).

As combinações de nucleosídeos análogos que mais demonstraram benefícios clínicos são zidovudina/ didanosina e zidovudina/zalcitabina. A combinação zidovudina/lamivudina pode ser mais bem tolerada e apresenta potência anti-retroviral comparável (Carpenter et al., 1996).

A terapia combinada tem se demonstrado mais eficaz que a monoterapia com AZT (Carpenter et al., 1996). A associação de 3TC e AZT mostrou sinergismo in vitro. $\mathrm{O} 3 \mathrm{TC}$ induz a resistência viral à ela própria, restituindo a sensibilidade ao AZT na transcriptase reversa em muitos pacientes que apresentam processo de resistência associada a zidovudina por meio de mutação, enfraquecendo a virulência e a capacidade de replicação viral do HIV1 e diminuindo a diversificação genética da enzima deste (Moore et al., 1999).

Este trabalho tem por objetivo avaliar o perfil farmacocinético, em plasma humano, dos anti-retrovirais lamivudina e zidovudina combinados em um único comprimido, através da obtenção dos parâmetros farmacocinéticos calculados estatisticamente e comparar estes resultados com a literatura consultada.

\section{MATERIAL E MÉTODOS}

\section{Critérios de seleção dos voluntários}

Após aprovação do protocolo de estudo pelo Comitê de Ética em Experimento Humano/UPE, 10 voluntários do sexo masculino com idade entre 19 e 37 anos (média 24 anos) e peso corporal entre 60 e $98 \mathrm{~kg}$ (média 74,7 kg) em consonância com a relação altura/peso, foram selecionados para o estudo de farmacocinética da formulação. Os voluntários foram submetidos a entrevista para esclarecimentos do protocolo de estudo, a exames clínicos e os escolhidos assinaram termo de consentimento.

$\mathrm{Na}$ avaliação clínica, os voluntários não apresentaram sinais ou sintomas evidentes de doenças cardíaca, hepática, renal, pulmonar, neurológica, gastrointestinal ou hematológica. Para tal constatação foi efetuada a anamnese dos voluntários, bem como foram avaliados os testes laboratoriais bioquímicos e hematológicos: glicemia, uréia, creatinina, TGO, TGP, gama GT, colesterol total, triglicerídeos, amilase e hemograma.

Foram excluídos todos os indivíduos em uso de qualquer medicamento, durante as 4 semanas anteriores ao teste, assim como indivíduos que houvessem participado de outro estudo nos últimos 3 meses ou apresentando sinais ou quadro comprovado de hepatopatia cirrótica ou ainda com neutropenia ou trombocitopenia moderadas ou graves. Foram, também, excluídos destes estudos voluntários fumantes (quinze ou mais cigarros por dia) e consumidores de bebidas alcóolicas (ingestão regular de mais de 4 unidades de álcool por dia: 1 unidade corresponde a $1 / 2$ garrafa de cerveja, 1 copo de vinho ou 1 dose de bebida destilada).

\section{Protocolo Clínico}

O ensaio farmacocinético da formulação foi realizado com administração de dose única de 2 comprimidos com $150 \mathrm{mg}$ de lamivudina e $300 \mathrm{mg}$ de zidovudina produzidos pelo Laboratório Farmacêutico do Estado de Pernambuco - LAFEPE. Os 10 voluntários foram internados por um período de $24 \mathrm{~h}$, sendo admitidos por volta de 10 horas do dia anterior ao início do estudo. Uma vez que a cinética de absorção de 3TC e AZT combinados não é alterada quando administrada junto a alimentos (Moore et 
al., 1999), os voluntários receberam café da manhã padrão e permaneceram sem ingerir nenhum tipo de alimentação após receberem a medicação. Os voluntários permaneceram internados por 12 horas após administração do medicamento e foram efetuadas coletas de sangue em intervalos de tempo regulares.

Uma hora após o café da manhã, foi realizada a primeira coleta $(\mathrm{t}=0)$ de aproximadamente $5 \mathrm{~mL}$ de sangue através de "butterfly" heparinizado, introduzido em uma veia superficial do antebraço do voluntário e coletado em tubos de ensaio heparinizados. Imediatamente após a primeira coleta foi feita a administração da formulação em teste em dose única com 2 comprimidos (ingeridos com $240 \mathrm{~mL}$ de água potável) e foram realizadas novas coletas de sangue nos seguintes intervalos de tempo: $10 \mathrm{~min}, 15$ min, 30 min, 45 min, 1 h, 2 h, 3 h, 4 h, 6 h, 8 h e 12 h.

As amostras de sangue foram imediatamente centrifugadas a $3000 \mathrm{~g}$ durante $10 \mathrm{~min}$, sendo então separado o plasma para armazenamento a $-20^{\circ} \mathrm{C}$ para posterior análise.

A dose utilizada foi baseada em trabalhos clínicos anteriores, respeitando-se os limites de segurança, toxidade e níveis plasmáticos justificáveis (Santos-Magalhães et al., 2000).

\section{Análise das amostras de plasma}

As amostras plasmáticas foram analisadas no Laboratório de Tecnologia Químico-Farmacêutica da Universidade Federal de Pernambuco, por Cromatografia Líquida de Alta Eficiência, utilizando-se metodologia analítica validada para esse estudo.

A curva de calibração foi preparada com seis níveis de concentração. Inicialmente, foi obtida solução mãe com concentrações de $100 \mu \mathrm{g} / \mathrm{mL}$ para o $3 \mathrm{TC}$ e $200 \mu \mathrm{g} / \mathrm{mL}$ para o AZT Desta solução foram preparados seis padrões com concentrações de $3,6,10,20,40$, e $70 \mu \mathrm{g} / \mathrm{mL}$ para $3 \mathrm{TC}$ e 6 , $12,20,40,80$ e $140 \mu \mathrm{g} / \mathrm{mL}$ para o AZT. Em microtubos foram acrescentados $450 \mu \mathrm{l}$ de plasma branco e em segui- da, para cada ponto da curva foi adicionada uma alíquota de $50 \mu \mathrm{L}$ do padrão correspondente (totalizando $500 \mu \mathrm{L}$ ) e $20 \mu \mathrm{L}$ do padrão interno $\delta$-4-oxietano (d4T) numa concentração de $100 \mu \mathrm{g} / \mathrm{mL}$. Os microtubos foram agitados em vortex até homogeneização. Adicionaram-se $100 \mu \mathrm{L}$ de ácido tricloroacético (20\%) e nova agitação em vortex para precipitação das proteínas plasmáticas. As amostras foram centrifugadas a $23.000 \mathrm{~g}$ durante 5 minutos sob refrigeração a $4{ }^{\circ} \mathrm{C}$. O sobrenadante foi recolhido e injetado no aparelho num volume de $20 \mu \mathrm{L}$. 3TC e AZT foram doseados simultaneamente em sistema cromatográfico constituído de cromatógrafo líquido de alta eficiência Waters (Miliford, MA, USA) com injetor automático conectado ao detector ultravioleta a $270 \mathrm{~nm}$. Os analitos foram separados em coluna Waters $\mathrm{C}_{18} \mu$ bondpack 300x3,9 mm ID com $10 \mu \mathrm{m}$ de tamanho de partícula e fase móvel isocrática constituída de $85 \%$ de tampão fosfato ( $\mathrm{pH} 5,6-0,05 \mathrm{M}$ ) e $15 \%$ de metanol, sob fluxo de $1,0 \mathrm{~mL} / \mathrm{min}$. As análises foram conduzidas a $25^{\circ} \mathrm{C} \pm 1{ }^{\circ} \mathrm{C}$ e os tempos de retenção foram de 8 minutos para o $3 \mathrm{TC}, 14$ minutos para o d4T e 27 minutos para o AZT. Os dados referentes aos ensaios de validação do método podem ser observados na Tabela I

Na preparação das amostras dos voluntários foram acrescentados $500 \mu \mathrm{L}$ de plasma e $20 \mu \mathrm{L}$ do padrão interno nos microtubos. A partir deste ponto, foi seguido o mesmo método analítico utilizado na obtenção da curva de calibração e todo o procedimento laboratorial foi conduzido segundo as boas práticas de bioequivalência e biodisponibilidade estabelecidas pela Agência Nacional de Vigilância Sanitária (ANVISA).

\section{Análise farmacocinética}

A análise dos resultados foi fundamentada nos cálculos da área parcial sob a curva de concentração em função do tempo $\left(\mathrm{ASC}_{0 \rightarrow 12}\right)$, da área total sob a curva de concentração em função do tempo $\left(\mathrm{ASC}_{0 \rightarrow \infty}\right)$, concentração máxima atingida $\left(\mathrm{C}_{\max }\right)$ e o tempo ao qual a mesma aparece $\left(\mathrm{T}_{\max }\right)$, constante de eliminação $\left(\mathrm{K}_{\mathrm{e}}\right)$, meia-vida de eli-

TABELA I - Dados referentes aos ensaios de validação do método cromatográfico de análise simultânea do 3TC e do AZT

\begin{tabular}{lcc}
\hline Parâmetro de validação & lamivudina & zidovudina \\
\hline Faixa de linearidade $(\mu \mathrm{g} / \mathrm{mL})$ & $0,3-7,0$ & $0,6-14,0$ \\
$\mathrm{R}^{2}$ & 0,9993 & 0,9988 \\
Precisão $(\%)$ & $2,13-4,81$ & $1,11-5,62$ \\
Exatidão $(\%)$ & $99,83-101,79$ & $98,15-100,85$ \\
$\mathrm{LD}(\mu \mathrm{g} / \mathrm{mL})$ & 0,07 & 0,08 \\
$\mathrm{LQ}(\mu \mathrm{g} / \mathrm{mL})$ & 0,15 & 0,13 \\
Recuperação $(\%)$ & 102,01 & 92,98 \\
\hline
\end{tabular}


minação $\left(\mathrm{t}_{1 / 2}\right)$, o clearence oral $\left(\mathrm{Cl}_{\mathrm{o}}\right)$ e volume aparente de distribuição $\left(\mathrm{V}_{\mathrm{d}}\right)$ a partir das formulações de 3TC/AZT do LAFEPE. O $\mathrm{C}_{\max }$ e o $\mathrm{T}_{\max }$, foram obtidos diretamente dos dados experimentais (Moore 1999 et al., Wang et al., 1994; Moore et al., 1996; Barry et al., 1996).

As áreas sob a curva observadas do tempo zero até 12 horas $\left(\mathrm{ASC}_{0 \rightarrow 12}\right)$ foram determinadas via cálculo numérico utilizando o método linear dos trapézios. A extrapolação desta área a um tempo infinito ( $\mathrm{ASC}_{0 \rightarrow \infty}$ ) foi estimada assumindo cinética de perfil exponencial após dose oral de formulação. Desta forma, $\mathrm{ASC}_{0 \rightarrow \infty}=\mathrm{ASC}_{0 \rightarrow 12}+\mathrm{C}_{\text {final }} / \mathrm{K}_{\mathrm{e}}$, onde $\mathrm{C}_{\text {final }}$ se refere a concentração do último ponto medido na curva plasmática concentração versus tempo. As constantes de eliminação terminal de primeira ordem $\left(\mathrm{K}_{\mathrm{e}}\right)$ foram estimadas a partir da equação de regressão linear entre o logaritmo natural da concentração e o tempo. As estimativas do tempo de meia-vida de eliminação $\left(\mathrm{t}_{1 / 2}\right)$ foram derivadas diretamente a partir desta constante de eliminação, empregando a equação $\mathrm{t}_{1 / 2}=\ln 2 / \mathrm{K}_{\mathrm{e}}$. Os valores de clearence oral $\left(\mathrm{Cl}_{\mathrm{o}}\right)$ foram obtidos pela razão entre a dose administrada e a $\mathrm{ASC}_{(0 \mathbb{})}$ e os volumes aparentes de distribuição foram obtidos pela razão entre o clearence oral e a constante de eliminação (Moore et al., 1999; Wang et al., 1999; Moore et al., 1996; Barry et al., 1994).

\section{RESULTADOS E DISCUSSÃO}

Após a administração dos comprimidos, os fármacos foram rapidamente absorvidos após a ingestão oral e bem tolerados pelos voluntários. Não foram observados efeitos adversos em nenhum voluntário durante o estudo, embora seja encontrado na literatura o relato de alguns efeitos sem grande relevância, após a administração oral desses dois anti-retrovirais. Os efeitos relatados foram: náusea, cefaléia, dor abdominal e fadiga (Van Leeuwen et al., 1992 e Johnson et al., 1999).

As concentrações plasmáticas médias em função do tempo de 3TC e AZT, observando-se os dez voluntários, podem ser analisadas na figura 3 e as médias geométricas com os respectivos desvios padrão dos parâmetros calculados dos dez voluntários são apresentados na tabela II.

\section{Perfil farmacocinético da lamivudina}

Considerando-se uma administração oral em dose única de dois comprimidos, perfezendo um total de 300 mg de 3 TC e $600 \mathrm{mg}$ de AZT, o $\mathrm{C}_{\text {max }}$ observado para o $3 \mathrm{TC}$ foi de $3,88 \mu \mathrm{g} / \mathrm{mL}$ junto a um $\mathrm{T}_{\text {max }}$ de 1,71 horas. Dados semelhantes são relatados por Johnson et al. (1999) e Santos-Magalhães et al. (2001), que realizaram um estudo similar com a 3TC isolado, bem como os resultados obtidos por Moore et al. (1999), observando os mesmos parâmetros com a 3TC associado ao AZT.

Os parâmetros dose dependentes de área sob a curva $\operatorname{ASC}_{0 \rightarrow \infty}\left(16,40 \mathrm{mg} \cdot \mathrm{h} \cdot \mathrm{mL}^{-1}\right), \mathrm{ASC}_{0 \rightarrow 12}\left(13,02 \mathrm{mg} \cdot \mathrm{h} \cdot \mathrm{mL}^{-1}\right) \mathrm{e}$ $\mathrm{C}_{\max }(3,88 \mu \mathrm{g} / \mathrm{mL})$ apresentaram-se um pouco elevados, comparando-se com os dados referenciados pela maioria dos autores citados anteriormente. Porém, os valores encontrados estão compatíveis com a dose administrada de 3TC (300 mg) e com os resultados obtidos por Moodley et al. (1998), Moore et al. (1999) e Santos-Magalhães et al. (2001).

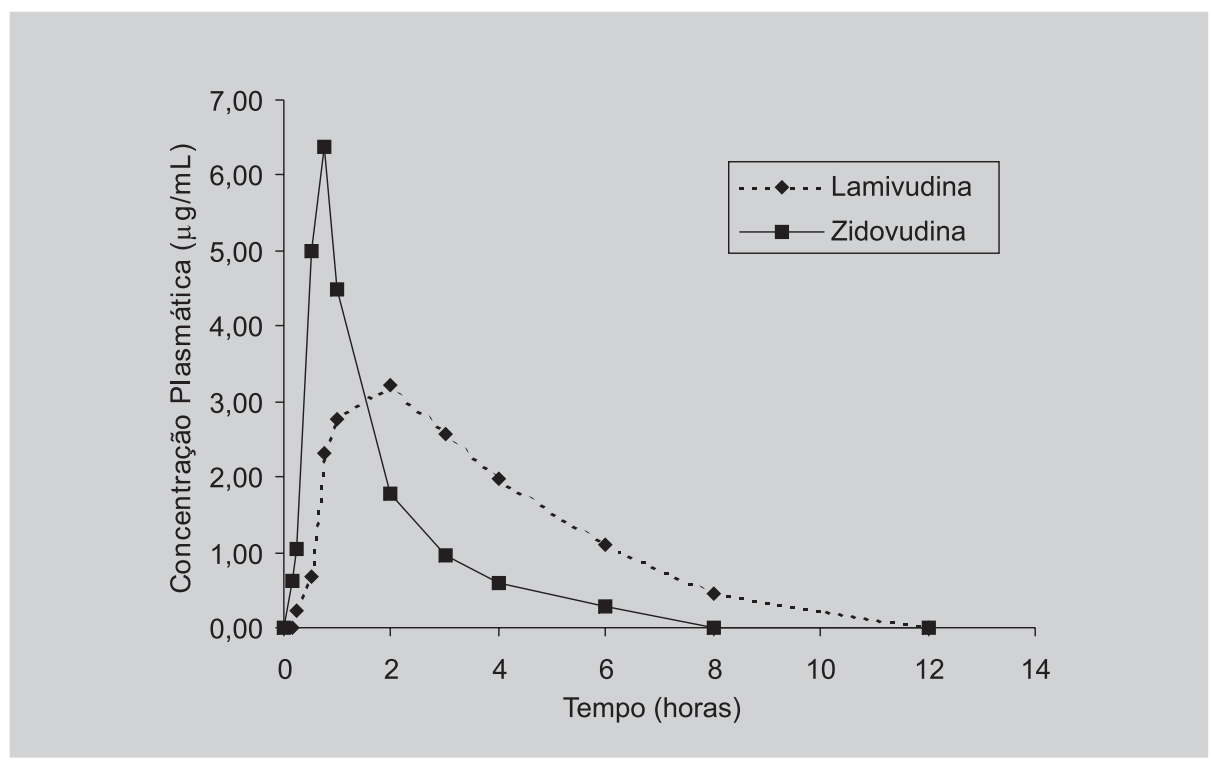

FIGURA 3 - Concentrações plasmáticas médias da lamivudina e da zidovudina. 
TABELA II - Parâmetros farmacocinéticos da lamivudina e da zidovudina para os 10 pacientes. Os resultados representam a média geométrica e os respectivos desvios padrão

\begin{tabular}{lcc}
\hline Parâmetros farmacocinéticos & lamivudina & zidovudina \\
\hline $\mathrm{K}_{\mathrm{e}}\left(\mathrm{h}^{-1}\right)$ & $0,27 \pm 0,06$ & $0,69 \pm 0,19$ \\
$\mathrm{t}_{1 / 2}($ horas $)$ & $2,57 \pm 0,62$ & $1,01 \pm 0,28$ \\
$\mathrm{ASC}_{0 \rightarrow \infty}\left(\mu \mathrm{g} \mathrm{h} \mathrm{mL}^{-1}\right)$ & $16,40 \pm 4,38$ & $9,80 \pm 2,77$ \\
$\mathrm{ASC}_{0 \rightarrow 12}\left(\mu \mathrm{g} \mathrm{h} \mathrm{mL}^{-1}\right)$ & $13,02 \pm 3,67$ & $8,86 \pm 2,47$ \\
$\mathrm{C}_{\max }\left(\mu \mathrm{g} \mathrm{mL} \mathrm{mL}^{-1}\right)$ & $3,88 \pm 1,20$ & $7,19 \pm 2,55$ \\
$\mathrm{~T}_{\text {max }}$ (horas) & $1,71 \pm 0,77$ & $0,71 \pm 0,14$ \\
$\mathrm{Cl}_{\mathrm{o}}(\mathrm{mL} / \mathrm{min})$ & $304,76 \pm 77,46$ & $1020,07 \pm 311,78$ \\
$\mathrm{~V}_{\mathrm{d}}(\mathrm{L} / \mathrm{kg})$ & $1,13 \pm 0,48$ & $1,49 \pm 0,56$ \\
\hline
\end{tabular}

Foi detectada uma constante de eliminação $\left(\mathrm{K}_{\mathrm{e}}\right)$ relativamente baixa $\left(0,27 \mathrm{~h}^{-1}\right)$. Este parâmetro pode ser explicado pelo fato do $3 \mathrm{TC}$ sofrer pouca metabolização hepática $(5$ a 10\%) e sua eliminação ser quase totalmente realizada através dos rins ( Perry, Fauldes, 1997; Johnson et al., 1999). A meia-vida de eliminação terminal estimada $\left(\mathrm{t}_{1 / 2}\right)$ de 2,57 horas ratifica os dados experimentais com um tempo de eliminação total de 12 horas, considerando-se que o $3 \mathrm{TC}$ foi completamente eliminado após 5 meias-vidas. Dados similares são citados por Van Leeuwen et al. (1992), num estudo de Fase I e por Santos-Magalhães et al. (2001), num estudo de bioequivalência, com o $3 \mathrm{TC}$ isolado.

Os valores calculados do volume aparente de distribuição $\mathrm{V}_{\mathrm{d}}$ e do clearence oral $\mathrm{Cl}_{\mathrm{o}}$ foram equiparáveis aos relatados por Moore et al. (1996, 1999). A partir da análise do volume aparente de distribuição $(1,13 \mathrm{~L} / \mathrm{kg})$ podese constatar a grande capacidade de penetração e distribuição do $3 \mathrm{TC}$ em todos os fluidos e tecidos do organismo, possivelmente, devido a fatores já descritos anteriormente como baixa massa molecular e pouca ligação às proteínas plasmáticas. Quanto à depuração do $3 \mathrm{TC}$, o clearence oral encontrado foi de $304,76 \mathrm{~mL} / \mathrm{min}$, indicando que sua eliminação é essencialmente renal.

\section{Perfil farmacocinético da zidovudina}

Após a administração oral dos comprimidos contendo os anti-retrovirais, a concentração sérica máxima foi alcançada 0,71 horas após a administração da dose. Os resultados indicam uma absorção rápida do AZT. Observa-se, também, eliminação rápida do AZT ( 8 horas). Estes resultados foram descritos pela maioria dos autores.

A concentração sérica máxima encontrada $(7,19 \mu \mathrm{g} / \mathrm{mL})$ mostrou-se um pouco superior em relação aos valores encontrados na literatura, porém, junto com os outros parâmetros dose dependentes $\operatorname{ASC}_{0 \rightarrow \infty}\left(9,80 \mu \mathrm{g} \mathrm{h} \mathrm{mL} L^{-1}\right)$ e
$\operatorname{ASC}_{0 \rightarrow 12}\left(8,86 \mu \mathrm{g} \mathrm{h} \mathrm{mL}^{-1}\right)$ mostraram se compatíveis com a dose administrada e com os resultados obtidos em estudos semelhantes realizados por Blum et al. (1988) Földes et al. (1993); Wang et al. (1999)

Com uma constante de eliminação $\left(\mathrm{K}_{\mathrm{e}}\right) 0,69 \mathrm{~h}^{-1}$ calcula-se a meia-vida terminal estimada $\left(\mathrm{t}_{1 / 2}\right)$ de 1,01 horas, muito pequena, evidenciando eliminação rápida e que o tempo de permanência da substância no plasma é curto. Estes parâmetros estão de acordo com os resultados previamente relatados na literatura para estudos de farmacocinética do AZT isolado (Blum et al., 1988; Földes et al., 1993; Wang et al., 1999) e compatíveis com estudo similar realizado com o AZT combinado com o 3TC (Moore et al., 1999).

O clearence oral $\left(\mathrm{Cl}_{\mathrm{o}}\right)$ e o volume aparente de distribuição $\left(\mathrm{V}_{\mathrm{d}}\right)$ calculados mostraram-se compatíveis com os resultados de Moore et al. (1999) e Wang et al. (1999). Observouse um volume aparente de distribuição do AZT de 1,49 L/kg, indicando grande penetração e distribuição da molécula nos fluidos e tecidos corpóreos e que a concentração de AZT no líquido extra vascular é aproximadamente $50 \%$ maior do que a concentração no plasma. Diferentemente do 3TC, o clearence oral foi alto $(1020,07 \mathrm{~mL} / \mathrm{min})$, sinalizando que a molécula de AZT sofre metabolização hepática.

\section{CONCLUSÃO}

De forma geral, os parâmetros dose-dependentes de ambas as substâncias mostraram-se um pouco elevados. Tais diferenças são oriundas, provavelmente, de variações na cinética de absorção entre os voluntários, possivelmente, de origem biológica, como peso, altura e teor de gordura, visto que o perfil fisiológico do homem adulto é diferente nas diferentes regiões do globo. Já os parâmetros independentes da dose apresentaram-se sempre dentro da faixa esperada, com resultados muito semelhantes aos encontrados na literatura consultada. 
Pode-se concluir que os resultados obtidos neste estudo estão compatíveis com o previsto na literatura. $\mathrm{O}$ fato de os anti-retrovirais estarem associados na mesma formulação não modificou o perfil cinético de nenhuma das substâncias, quando comparam-se os parâmetros com os citados por outros autores em trabalhos semelhantes.

\section{AGRADECIMENTOS}

Agradecemos ao Departamento de Ciências Farmacêuticas - UFPE e ao CNPq, pelo suporte financeiro parcial.

\section{ABSTRACT \\ Pharmacokinetic evaluation of lamivudine and zidovudine tablets in human plasma}

The purpose of this work was an evaluation of the pharmacokinetic profile from lamivudine and zidovudine combination tablets, produced by Laboratório Farmacêutico do Estado de Pernambuco - LAFEPE. The formulation was administered in 10 volunteers and the concentration-time serum profile was observed from both antiretroviral agents during 12 hours. From concentration-time serum profile the pharmacokinetic parameters for both drugs were calculated by statistical methods. The results were compared with those from experiments carried out by other authors referred in this paper. Differences between the results obtained herein and those from other authors were not significant. The kinetic profile for both substances was not modified by the antiretroviral combination.

UNITERMS: Pharmacokinetic. Lamivudine. Zidovudine.

\section{REFERÊNCIAS BIBLIOGRÁFICAS}

BARRY, M.; HOWE, J. L.; ORMESHER, S.; BACK, D. J.; BRECKENRIDGE, A. M.; BERGIN, C.; MULCAHY, F.; BEECHING, N.; NYE, F. Pharmacokinetics of zidovudine and dideoxyinosine alone and in combination in patients with the acquired immunodeficiency syndrome. Br. J. Clin. Pharm., v. 37, p. 421-26, 1994.

BLANEY, S. M.; DANIEL, M. J.; HARKER, A J.; GODWIN, K.; BALIS, F. M. Pharmacokinetics of lamivudine and BCH-189 in plasma and cerebroespinhal fluid of nonhuman primates. Antimicrob. Agents Chemother., v. 39, n.12, p. 2779-2782, 1995.
BLUM, M. R.; LIAO, S. H.; GOOD, S. S.; DE MIRANDA, P. Pharmacokinetics and bioavailability of zidovudine in humans. Am. J. Med., v. 85, n. 2A, p. 189-94, Aug 1988.

CARPENTER, C. C.; FISCHL, M. A.; HAMMER, S. M.; HIRSCH, M. S.; JACOBSEN, D. M.; KATZENSTEIN, D. A.; MONTANER, J. S.; RICHMAN, D. D.; SAAG, M. S.; SCHOOLEY, R. T.; THOMPSON, M. A.; VELLA, S.; YENI, P. G.; VOLBERDING. P. A. Antiretroviral therapy for Infection in 1996. J. Am. Med. Assoc. JAMA, v. 276, n. 2, p. 146-54, 1996.

FÖLDES, O.; UHEROVA P.; MAYER, V. Plasma levels of the anti-HIV drug 3'-azido-2',3'-dideoxythymidine (AZT): determination by RIA and HPLC. Acta Virol., v. 37, n. 2-3, p. 156-64, 1993.

GOOD, S. S.; DURACK, D. T.; DE MIRANDA, P. Biotransformation in various species and in humans of 3'-azido-3'-deoxy-thymidine, a potential agent for the treatment of AIDS. Fed. Proc. v. 45, n. 3, p. 444, 1986.

HEALD, A. E.; HSYU, P. H.; YUEN, G. J.; ROBINSON, P.; MYDLOW, P.; BARTLETT, J. A. Pharmacokinetics of human immunodeficiency virus-infected patients with renal disfunction. Antimicrob. Agents Chemother., v. 40, p. 1514-1519. 1996.

HEDAYA, M. A.; SAWCHUK, R. J. A sensitive liquidchromatographic method for determination of 3'-azido3 'deoxythymidine (AZT) in plasma and urine. Clin. Chem., v. 34, n. 8, p. 1565-68, 1988.

HENRY, K.; CHINNOCK, B. J.; QUINN, R. P.; FLETCHER, C. V.; DE MIRANDA, P.; BALFOUR Jr., H. H.. Concurrent zidovudine levels in semen and serum determined by radioimmunoassay in patients with AIDS or AIDS-related complex. J. Am. Med. Assoc. JAMA, v. 259, p. 3023-26, 1988.

JOHNSON, M. A.; MOORE, K. H.; YUEN, G. J.; BYE, A.; PAKES, G. E. Clinical pharmacokinetics of lamivudine. Clin. Pharmacokinet., v. 36, n. 1, p. 41-66, 1999.

KLECKER Jr., R. W.; COLLINS, J. M.; YARCHOAN, R.; THOMAS, R.; JENKINS, J. F.; BRODER, S.; MYERS, C. E.Plasma and cerebrospinal fluid pharmacokinetics of 3'-azido-3'-deoxythymidine: a novel pyrimidine analog with potential application for the treatment of patients with AIDS and related diseases. Clin. Pharmacol. Ther., v. 41, n. 4, p. 407-12, 1987. 
LANGTRY, H. D.; CAMPOLI-RICHARDS, D. M. Zidovudine. A review of its pharmacodynamic and pharmacokinetic properties, and therapeutic effcacy. Drugs, v. 37. p. 408-50, 1989.

LARDER, B. A.; KEMP, S. D.; HARRIGAM, P. R. Potential mechanism for sustained antiretroviral efficacy of AZT3TC combination therapy. Science, v. 269, p. 696-99, 1995.

LEWIS, L. L.; VEZON, D.; CHURCH, J. Lamivudine in children with human immunodeficiency virus infection: a phase I/II study. J. Infect. Dis., v. 174, p. 16-25, 1996.

MOODLEY, J.; MOODLEY, D.; PILLAY, K.; COOVADIA, H.; SABA, J.; VAN LEEUWEN, R.; GOODWIN, C.; HARRIGAN, P. R.; MOORE, K. H.; STONE, C.; PLUMB, R.; JOHNSON, M. A. Pharmacokinetics and antiretroviral activity of lamivudine alone or when coadministered with zidovudine in human immunodeficiency virus type 1 -infected pregnant women and their offspring. J. Infect. Dis., v. 178, n. 5, p. 1327-33, 1998.

MOORE, K. H.; YUEN, G. F.; RAASCH, R. H.; ERON, J. J.; MARTIN, D.; MYDLOW, P. K.; HUSSEY, E. K. Pharmacokinetics of lamivudine administered alone and with trimethoprim-sulfamethoxazole. Clin. Pharmacol. Therap., v.59, n. 5, p.550-558, 1996.

MOORE, K. H.; SHAW, S.; LAURENT, A. L.; LLOYD, P.; DUNCAN, B.; MORRIS, D. M.; O'MARA, M. J.; PAKES, G. E. Lamivudine/zidovudine as a combined formulation tablet: bioequivalence compared with lamivudine and zidovudine administered concurrently and the effect of food on absorption. J. Clin. Pharmacol., v.39, n. 6, p. 593-605, 1999.

PERRY, C M.; FAULDES, D. A review of its antiviral activity, pharmacokinetic properties and therapeutic efficacy in the management of HIV infection. Drugs, v. 53, p. 657-680, 1997.
ROBBINS, B. L.; TRAN, T. T.; PINKERTON, F. H. Jr.; AKEB, F.; GUEDJ, R.; GRASSI, J.; LANCASTER, D.; FRIDLAND, A. Development of a new cartridge radioimmunoassay for determination of intracellular levels of lamivudine triphosphate in the peripheral blood mononuclear cells of human immunodeficiency virusinfected patients. Antimicrob. Agents Chemother., v. 42, n. 10, p. $2656-60,1998$.

SANTOS-MAGALHAES, N. S.; PONTES, A.; CAVALCANTE, R. M.; COSTA, R. M.; RANGEL, F. A.; GUIMARAES, M. I.; DE CARVALHO J. N.; DE SOUZA, S. D.; DE OLIVEIRA, H. M.; ESTEVES, I. L.; RAMALHO, M. S.; VIEIRA, S. L.; ALVES, A. J. Bioequivalence of two lamivudine tablets. ArneimittelForschung. Drug Res., v. 51, n. 04, p. 1-10, 2001.

SLUSHER, J. T.; KUWAHARA, S. K.; HAMZEH, F. M.; LEWIS, L. D.; KORNHAUSER, D. M.; LIETMAN, P. S. Intracellular zidovudine (ZDV) and ZDV phosphates as measured by a validated combined high-pressure liquid chromatography-radioimmunoassay procedure. Antimicrob. Agents Chemother., v. 36, n.11, p. 2473-7, 1992.

VAN LEEUWEN, R.; KATLAMA, C.; KITCHEN, V. Evaluation of safety efficacy of 3TC (Lamivudine) in patients with asymptomatic or mildly symptomatic human immunodeficiency virus infection: a phase I/II study. J. Infect. Dis., v. 171, p. 1166-71, 1995.

VAN LEEUWEN, R.; LANGE, J. M.; HUSSEY, E. K.; DONN, K. H.; HALL, S. T.; HARKER, A. J.; JONKER, P.; DANNER, S. A. The safety and pharmacokinetics of a reverse transcriptase inhibitor, 3TC, in patients with HIV infection: a phase I study. AIDS., v. 6, n. 12, p.14715,1992 .

WANG, L. H.; CHITTICK, G. E.; McDOWELL, J. A. Single-dose pharmacokinetics and safety of abacavir (1592U89), zidovudine, and lamivudine administered alone and in combination in adults with human immunodeficiency virus infection. Antimicrob. Agents Chemother., v. 43, n. 7, p. 1708-15, 1999.

Recebido para publicação em 05 de abril de 2003. 